\title{
LOS JÓVENES CIUDADANOS: REFLEXIONES PARA UNA POLÍTICA DE FORMACIÓN CIUDADANA JUVENIL *
}

\author{
M. LORETO MARTÍNEZ \\ CARMEN SILVA** \\ MARGARITA MORANDÉ \\ LILIAN CANALES ${ }^{* * * *}$
}

\begin{abstract}
RESUMEN
Este artículo analiza los resultados de estudios previos sobre las nociones de ciudadanía y aspiraciones de ejercicio ciudadano que tienen jóvenes chilenos, y la manera en que están siendo abordados por los programas existentes en nuestro país. La presentación identifica los desafíos que se plantean para el desarrollo de una política educacional como contexto macrosocial que promueva la formación ciudadana. Se analizan también aspectos más específicos de la formación ciudadana que se plantean en los programas existentes: a) creación de una cultura escolar democrática; b) valoración de la formación ciudadana y el rol de los adultos; c) sintonía entre el programa, proyecto educativo y entorno institucional; d) fortalecimiento de las metodologías y equipos ejecutores; e) problematización de la pobreza; y f) extensión de estas experiencias a los contextos de vida reales de los jóvenes. Se analizan las implicancias para el fortalecimiento del trabajo educativo en el contexto escolar y una política de formación ciudadana juvenil.
\end{abstract}

\section{PALABRAS CLAVE: CIUDADANÍA, JUVENTUD, PROGRAMAS DE FORMACIÓN CIUDADANA}

* $\quad$ Este trabajo fue realizado como parte del Proyecto Fondecyt 1085231 «Desarrollo cívico en jóvenes chilenos».

** Doctora en Psicología, Profesora Escuela de Psicología, Pontificia Universidad Católica de Chile. Correo electrónico:mlmartig@uc.cl.

*** Magíster en Psicología, Escuela de Psicología, Pontificia Universidad Católica de Chile. Correo electrónico: cusilva@uc.cl.

**** Magíster en Psicología, Escuela de Psicología, Pontificia Universidad Católica de Chile. Correo electrónico: mmorande@uc.cl.

***** Psicóloga, Escuela de Psicología, Pontificia Universidad Católica de Chile. Correo electrónico: lacanale@uc.cl. 


\title{
JOVENS CIDADÃOS: REFLEXÕES PARA. UMA POLÍTICA DE FORMAÇÃO CIDADÃ JUVENIL
}

\begin{abstract}
RESUMO
Este artigo analisa os resultados de estudos anteriores sobre as noções de cidadania e aspirações de exercício cidadão de jovens chilenos, bem como a maneira que estão sendo abordadas pelos programas existentes em nosso país. O artigo identifica os desafios que se colocam para o desenvolvimento de uma política educacional como contexto macrossocial que promova a formação cidadã. São analisados também aspectos mais específicos da formação cidadã que fazem parte dos programas existentes tais como: a) criação de uma cultura escolar democrática; b) valorização da formação cidadã e o papel dos adultos; c) sintonia entre o programa, projeto educativo e entorno institucional; d) fortalecimento das metodologias e equipamentos executores; e) problematização da pobreza; e f) extensão destas experiências aos contextos de vida reais dos jovens. São analisadas as implicações para o fortalecimento do trabalho educativo neste contexto escolar e uma política de formação cidadã juvenil.
\end{abstract}

PALAVRAS-CHAVE: CIDADANIA, JUVENTUDE, PROGRAMAS DE FORMAÇÃO CIDADÃ

\section{YOUTH CITIZENS: REFLEXIONS ON A CITIZEN YOUTH TRAINING POLICY}

\begin{abstract}
This article analyzes the results of previous studies on the concepts of citizenship and civic exercise aspirations of young Chileans who have, and how these are being addressed by existing programs in our country. The presentation identifies the challenges for the development of education policy and macro-social context that promotes civic education. An analysis is also more specific aspects of citizenship education that arise in existing programs such as: a) creation of a democratic school culture, b) an assessment of citizenship education and the role of adults; c) line between the program, educational project and institutional environment; d) strengthening of the methodologies and equipment implementing e) problematization of poverty; f) extension of these experiences in real life contexts of young people. We analyze the implications for strengthening the educational work in the school and a youth civic education policy.
\end{abstract}




\section{INTRODUCCIÓN}

EL CONCEPTO «CIUDADANÍA» TIENE significados teóricos que se plasman en variadas definiciones, entre las que destacan elementos como «pertenecer a cierta comunidad política nacional» (Janoski \& Gran, 2002:15-16; Martínez, 1999:11-12; Turner, 1993:9-10), «poseer derechos civiles, políticos y sociales» (CEPAL, 2000; Martínez, 1999), «tener capacidad de influencia en políticas tendientes a la igualdad» (Janoski \& Gran, 2002:30-32) y «cumplir ciertos deberes y responsabilidades en la conducción de la vida común» (CEPAL, 2000:302-304).

Además de un concepto, la ciudadanía también es una práctica; es decir, una forma de actuar que se construye a través de las experiencias de participación en la sociedad, que se reproduce en los espacios sociales y políticos y que se representa en el espacio social intersubjetivo (González, 2007:337-338). Por tanto, su estudio debe ser contextualizado sociohistóricamente (Bárcena, 1997) ya que se halla en permanente evolución, y su ejercicio y representación pueden tomar diferentes formas en distintos actores e instituciones de la sociedad, con mayor o menor énfasis en diversos aspectos de este constructo.

En este artículo nos centramos en los jóvenes como actores del proceso democrático y en la manera en que las instituciones que participan de su educación (e.g., familia, escuela, organizaciones comunitarias, medios de comunicación social) pueden intencionar su actuación en tanto sujetos participativos y con voz al interior de la sociedad civil.

Los hallazgos de estudios previos (González, 2007:335-372; Martínez, Silva \& Hernández, en revisión; Martínez, Silva, Carmona, Cumsille \& Flanagan, en revisión) sobre las creencias y aspiraciones en este ámbito que tienen los jóvenes chilenos indican que poseen una visión y aspiraciones de ciudadanía, y que se comprometen en diferentes tipos de participación social; o sea, un retrato distinto de la supuesta apatía por asuntos cívicos que se les ha atribuido a partir de las cifras de baja inscripción electoral.

Por ejemplo, los resultados de dos estudios cualitativos (el primero realizado con 24 jóvenes de 16 a 25 años que participaban en diferentes tipos de organizaciones formales e informales (política, voluntariado social, étnico-política, ecológica y estudiantil) y de condición socioeconómica diversa, y el segundo, en una muestra de 71 personas con características sociodemográficas similares pero que incluía a participantes y no participantes en organizaciones sociales) indican que los jóvenes perciben fuertes contradicciones entre un discurso 
«ideal» de ser ciudadano y las oportunidades reales que el sistema político y económico actual brinda a las personas, particularmente a los jóvenes, para ejercer una participación ciudadana efectiva. En su visión, la democracia no funciona bien, a diferencia de lo que perciben en el discurso de los adultos, particularmente aquel que se difunde en los medios de comunicación social.

Los resultados de estos estudios indican que los jóvenes relevan tres aspectos centrales en la ciudadanía:

i) La pertenencia a un grupo, sociedad o a un país. Los jóvenes refieren prejuicio y discriminación hacia ellos, su imagen y su cultura, situación potenciada por los medios de comunicación que difunden aspectos negativos de las movilizaciones que efectúan, sin valorar la contribución positiva de su acción. Los jóvenes aspiran a una cultura comunitaria que valore la pertenencia.

ii) Los derechos económicos y sociales, como la equidad de oportunidades (sin discriminación económica) en el acceso a educación, salud, vivienda, justicia, seguridad ciudadana, recreación, entre otros, que signifiquen verdaderamente libertad de elegir y respeto a los derechos sociales. Desde esta perspectiva, los jóvenes denuncian la discriminación existente y la hegemonía del dinero para determinar las oportunidades y la vulneración de derechos en algunos grupos de la sociedad. Perciben clases sociales marcadas que conllevan creencias de superioridad o privilegios del adinerado, vulnerando los derechos de aquellos que se encuentran en situación de pobreza (e.g. jornadas de trabajo y remuneraciones).

iii) Opinar, disentir, tomar decisiones y participar en el logro de metas comunes, generando cambios a través de la participación. Concordante con los hallazgos de González (2007), los jóvenes adscriben a una ciudadanía activa que valora la responsabilidad de informarse, la deliberación de los ciudadanos y la participación en la toma de decisiones, con voz en asuntos políticos y poder de influencia a diferentes niveles del agregado social. Ellos manifiestan la importancia de que su voz sea tomada en cuenta y rescatan la importancia de mantener la capacidad de lucha participando en organizaciones; a vía de ejemplo mencionan la «revolución de los pingüinos», ${ }^{1}$ como una búsqueda emblemática de igualdad en la calidad de la educación. La moviliza-

1 Movimiento de estudiantes secundarios durante el año 2006, dirigido a obtener importantes reformas en el sistema educacional actual. 
ción de los estudiantes visibilizó el problema de la calidad de la enseñanza en la agenda pública y se escucharon inicialmente sus demandas, pero, en su visión, este proceso los excluyó en la toma de decisiones. Los jóvenes reclaman la falta de poder real de los ciudadanos, particularmente del segmento joven y de aquellos económicamente desaventajados. Así, una gran deuda pendiente es la participación influyente, cuyo objetivo debe ser la defensa de los intereses de la mayoría en la sociedad y la búsqueda de equidad social.

En conjunto, los hallazgos analizados son muy consistentes con los de González (2007:367-368), basados en un análisis comparativo de los resultados del Cuestionario Res-Civitas y de la evaluación de las competencias cívicas realizada por la International Association for the Evaluation of Educational Achievement (IEA) (Torney-Purta, Lehmann, Oswald, \& Schulz, 2001:22-24) en una muestra representativa de estudiantes chilenos (MINEDUC, 2005:22-24). Estos indican que, para los jóvenes, el concepto de ciudadanía recoge una aspiración por la igualdad de acceso e integración social. Asimismo, confirma la percepción de los jóvenes de no ser considerados o entendidos por el mundo adulto, y su aspiración de una ciudadanía plena que los refleje e integre también a ellos. Esta percepción se correlaciona con la visión optimista de atribuirse posibilidades de éxito en los proyectos de vida, a través del esfuerzo y los objetivos que se han propuesto, valorando las oportunidades y los derechos civiles, políticos, económico-sociales y culturales existentes. La pobreza también es vista como «una carencia objetiva asociada a una pérdida en la integración ciudadana».

Respecto a los derechos políticos, González (2007:367) señala que el menor interés de los jóvenes se debe a «una desconfianza y deslegitimación de la institucionalidad de la política y la formalidad de los procedimientos democráticos» (2007:364) que, sin embargo, no refiere a un desinterés por lo político o lo público. Asimismo, existe concordancia entre los resultados de los estudios respecto a la participación, en el sentido de que los jóvenes valoran mucho más la direccionalidad hacia fines inmediatos, en correspondencia con la «problemática directa y personal de los individuos».

Los hallazgos también revelan la valoración de los derechos económico-sociales y culturales, el acceso a una efectiva igualdad de oportunidades, la no discriminación (e.g., de su condición de juventud y de los sectores desventajados económicamente) como camino de integración social y superación de la exclusión. Para los jóvenes, la pertenencia 
se asocia a «referentes que traduzcan un vínculo social pertinente a sus intereses y aspiraciones» (p.365). Los resultados, a la vez, coinciden en que ellos manifiestan una orientación a la ciudadanía activa, una «intención del ejercicio de la ciudadanía como titularidad de derechos y como pertenencia activa a una comunidad reflexiva y con tendencia a la acción en pro de sus intereses y de la ampliación de sus derechos» (p.365), en función de sus proyectos de vida, la apropiación de su futuro, y un posicionamiento próximo en el mundo adulto.

Las visiones y aspiraciones de ciudadanía de los jóvenes descritas anteriormente sugieren problemas de diferente orden, desde cambios en el sistema político hasta prácticas de convivencia a diferente nivel del agregado social. Y mueven a pensar la formación en ciudadanía de la juventud y sus implicancias para la formulación de una política social en el ámbito educacional y comunitario que incorpore la contribución de los jóvenes a la renovación de la sociedad. Asimismo, su visión plantea desafíos para los programas y contextos de formación ciudadana y su efectividad, en función del desarrollo de los jóvenes y del proceso de consolidación democrática en Chile.

Este artículo aborda los desafíos para la política educacional y para una política de formación ciudadana, a partir tanto de la visión de los jóvenes como de la información relativa a programas de formación ciudadana existentes en nuestro país.

\section{DESAFÍOS PARA LA POLÍTICA EDUCACIONAL COMO CONTEXTO MACROSOCIAL DE LA FORMACIÓN CIUDADANA}

Entre los desafíos pendientes de abordar en las políticas sociales en nuestro país destaca la segregación económica e inequidad entre la calidad de la educación pública y privada en Chile, condición que limita estructuralmente el potencial de la formación ciudadana para obtener o mantener resultados positivos. La segregación económica y espacial de los establecimientos educacionales y la desvalorización de la calidad de educación que se imparte en aquéllos del sector público dificultan los procesos de identificación y pertenencia de los estudiantes con la institución educativa, a la vez que crean sentimientos de discriminación y desesperanza en relación con su potencial logro educacional, a su valor como personas y a su proyecto futuro.

Un desafío mayor vinculado a la inequidad es promover la formación ciudadana en contextos de elevada vulnerabilidad social (e.g., pobreza, capital cultural disminuido), barrios y/o escuelas en las cuales se 
ha instaurado la violencia o la dominación de parte de ciertos grupos como forma habitual de relación. La inversión en recursos humanos para revertir estas culturas debe ser mayor y los objetivos de los programas deben considerar específicamente las características culturales y la realidad social de los participantes. En algunos casos, debe priorizarse la formación en habilidades sociales y de comunicación interpersonal como requerimientos básicos para la formación ciudadana.

En algunos de estos contextos, formar en ciudadanía puede resultar, en cierta medida, contracultural. En muchos casos se trata de contextos con valores y normas que no acogen bien la aplicabilidad de estas competencias. Por ejemplo, sustentar un lenguaje de derechos puede incomodar a adultos, o bien, los mismos jóvenes pueden no entender el desarrollo de una relación horizontal. Puede suceder también que el contexto escolar no sea democrático y el programa de formación ciudadana intente serlo, o la cultura escolar sea restrictiva e inhibidora, lo que representa un desafío para el logro de los objetivos que se proponen los programas, sobre todo si no se considera un trabajo con los demás actores de la comunidad escolar y, en muchos casos, con la familia y la vecindad.

Asimismo, el sentimiento de discriminación y estigmatización que perciben los jóvenes, especialmente aquellos provenientes de sectores vulnerables socialmente, de parte del mundo adulto resulta contracultural, pues limita los objetivos de promover mayor inclusión y desarrollar competencias participativas para el ejercicio ciudadano. En síntesis, la inequidad y aspectos asociados contradicen la base de la ciudadanía, por lo que dificultan el logro de objetivos de los programas de formación ciudadana.

Para acoger las propuestas de los jóvenes es urgente reforzar políticas de equidad y no discriminación a la cultura juvenil, visibilizando fuertemente sus fortalezas y contribución social, cuanto en sus estilos, tanto en su participación social y ciudadana, ya que aun en sus expresiones de protesta hay valores democráticos, de equidad, etcétera, los cuales debieran ser visibilizados por los medios de que dispone la administración democrática.

a) Participación real y efectiva en un contexto educativo democrático

Respecto a la gestión de las escuelas, las competencias ciudadanas se incrementan significativamente en aquellos establecimientos con pro- 
yectos educativos claramente perfilados (Comisión convocada por el Ministerio de Educación en Chile, 2004:62-64). Aquellos establecimientos que practican una gestión institucional de mayor horizontalidad, con instancias de participación docente claras y expeditas y donde se advierte un sentido de identidad y pertenencia claramente desarrollado, facilitan el logro de habilidades ciudadanas democráticas con los alumnos.

Se reconoce que, además, estas prácticas deben ser apoyadas desde los distintos niveles del proceso educativo; es decir, desde el apoyo y lineamientos que establezca el Ministerio de Educación en el país, hasta aquellos actores que tengan una relación directa con los niños y jóvenes. Así, por ejemplo, en nuestro país la formación ciudadana no posee una posición clara en el currículo y tiene que ser sacrificada frente a otros contenidos de carácter prioritario (e.g., ciencias y matemáticas), y esta situación también se refleja en la escasa medición de estas competencias en otros instrumentos (SIMCE), recursos asignados, indicadores de desempeño y estatus para el establecimiento educacional. Asimismo, estas habilidades y conocimientos no son medidos en las pruebas regulares de calidad de la educación de la nación.

\section{b) Evaluación de impacto}

Uno de los desafíos que presenta el desarrollo de competencias ciudadanas es el de establecer en qué momento y a través de qué indicadores se deben evaluar sus resultados y su impacto. Las competencias ciudadanas comprenden una integración compleja de distintos aspectos: conocimientos, habilidades y actitudes relacionadas a ésta, lo cual no siempre se manifiesta de forma inmediata. Asimismo, los canales de participación cívica variarán según la etapa evolutiva de las personas, por lo que resulta importante hacer un seguimiento posterior de los alcances a largo plazo de la formación en los individuos.

\section{DESAFÍOS PARA LA POLÍTICA DE PROGRAMAS DE FORMACIÓN CIUDADANA}

A partir de los estudios sobre las perspectivas de ciudadanía de los jóvenes y de las características de los programas de formación ciudadana existentes se discutirán algunos desafíos para estos últimos. 
a) Creación de una cultura escolar democrática que permita generar sentido de identidad y membresía

El proceso de socialización de niños y jóvenes está enraizado en las experiencias de membresía en las instituciones de su comunidad/sociedad, en el ejercicio de derechos y en el cumplimiento de responsabilidades que posibilitan la integración social. A través de las prácticas en las instituciones, los niños aprenden las reglas del orden social y a razonar acerca de ellas. Del análisis y experiencias con otros, los jóvenes desarrollan teorías, políticas y aspectos cognitivo-afectivos propios de la formación ciudadana. Las prácticas de estas organizaciones moldean las disposiciones de los jóvenes y de sus pares.

\section{b) Valoración de la formación en ciudadanía y rol de los adultos como facilitadores}

El aprendizaje de actitudes políticas y los compromisos para con los demás se hallan anclados en las relaciones sociales; esta vinculación resulta central para la generación de programas efectivos. De esta manera, los programas efectivos deben crear esta vinculación. Así, se hace necesario el establecimiento de un clima que propicie los valores ciudadanos (respeto, aceptación, expresión, etcétera), modelando la conducta de los niños y jóvenes e incoando un espacio apto para el aprendizaje. Por ejemplo, se reconoce la necesidad de que el profesor genere un ambiente interactivo dentro del aula y promueva un proceso de enseñanza acorde a los principios democráticos y de participación. Así, los adultos cumplen un rol clave como promotores de un ethos cívico (Flanagan et al., 1998:460-62).

\section{c) Sintonía entre el programa, el proyecto educativo y el entorno institucional}

El alcance de los programas puede verse obstaculizado si la formación que reciben los niños y jóvenes resulta contracultural con los predicamentos de una institución escolar o una comunidad que castiga la participación, la toma de conciencia, o la libertad de expresión. O bien, si la institución educacional y/o los adultos o figuras de autoridad (e.g., educadores) son desvalorizados o estigmatizados. En todos estos casos, la transmisión de valores y/o los procesos e identificación positiva se obstaculizarán. 
d) Fortalecimiento de los equipos ejecutores e implementación de metodologías acordes a la complejidad de estas competencias

En nuestro país, la escasez de tiempo que poseen los docentes para implementar estos programas (que descansan muchas veces en su buena voluntad y dedicación fuera del horario escolar), además del escenario escolar con múltiples contingencias y demandas (efemérides) reducen el tiempo para la implementación real de los programas de formación ciudadana.

Como resultado de esta realidad, se evidencian equipos sobreexigidos y que no siempre cuentan con el involucramiento institucional en la formación ciudadana. Asimismo, esto suscita una interrogante respecto de la posibilidad de masificar este tipo de formación, más allá de quienes, tanto profesores como alumnos, muestran un interés destacado por ello.

\section{e) Problematización de la pobreza}

Algunos programas enfatizan la promoción de derechos sociales, y muchos sitúan su accionar en sectores vulnerables. Sin embargo, cabe preguntarse si contemplan contenidos asociados a estas problemáticas: comprensión y análisis del modelo económico, de las políticas sociales, la legislación social (e.g., el Código del Trabajo, las políticas distributivas, etcétera). El análisis y la reflexión crítica de experiencias resultan centrales en el desarrollo de los ciudadanos. Para que los jóvenes puedan participar en el ámbito público es importante abordar contenidos como la institucionalidad política actual con sus respectivos mecanismos de representación, la democracia representativa versus participativa, los mecanismos democráticos en otras latitudes y los sistemas de control ciudadano, entre otros. En definitiva, estos conocimientos les permitirán comprender y encauzar sus críticas acerca de lo que ven y viven, para así desarrollar a futuro propuestas tendientes a superar los problemas sociales.

\section{f) Extensión de las experiencias de participación real e influyente a los contextos de vida reales de los jóvenes}

Las competencias ciudadanas comprenden un conjunto de conocimientos y de habilidades cognitivas, emocionales y comunicativas que, articulados entre sí, hacen posible que el ciudadano actúe de ma- 
nera constructiva en la sociedad democrática (MINEDUC, 2005:15-19) $\mathrm{y}$, a su vez, incremente sus competencias a través de sus experiencias de participación. El desarrollo ciudadano es un proceso de vida tanto personal como social, y se puede fortalecer a través de políticas y procesos a diferente nivel. En muchos casos, éstos implican tiempos de largo alcance, lo que hace necesario considerar los recursos humanos y económicos correspondientes para lograr los objetivos. Por ello, resulta relevante identificar y potenciar las oportunidades para que los niños aprendan temprano y gradualmente, de acuerdo a sus capacidades evolutivas, los componentes afectivos, cognitivos, sociales y comportamentales de la ciudadanía.

Los resultados de los estudios permiten no sólo formular desafíos para una política juvenil, sino también reflexionar acerca de la capacidad de los programas para motivar a los jóvenes. En lo fundamental, preguntarse si la manera en que se está abordando la formación ciudadana en Chile contribuye realmente a despertar en niños y jóvenes el interés por ser ciudadanos activos.

La formación de actitudes y habilidades ciudadanas tiene que ser anclada en las experiencias cotidianas de niños y jóvenes. Un contexto ideal para la formación en esos aspectos es el colegio. En Chile, la Formación Ciudadana de niños, niñas y jóvenes se contempla en la educación formal, tanto en el ciclo de enseñanza básica como media, cristalizada en los Objetivos Fundamentales Transversales del currículo oficial del MINEDUC. No obstante, las formas de llevar a cabo estos objetivos difieren en los distintos establecimientos.

En línea con este currículo, o en forma complementaria, hay programas de instituciones particulares y de entidades que trabajan en forma coordinada con organismos públicos como el Ministerio de Educación y el Servicio Nacional de Menores (Programas de Prevención Comunitaria). También existen escuelas y/o colegios (particulares o públicos) que explicitan dentro de su proyecto educativo el desarrollo de estas competencias, o bien que trabajan intencionadamente dentro de sus actividades, ciertos contenidos, habilidades o actitudes propias de la formación ciudadana.

$\mathrm{Al}$ revisar los programas existentes cabe señalar que algunos ponen el acento en:

i) La deliberación, la argumentación, procesamiento de la información utilizando la temática legislativa y representativa. Así, algunos programas enfatizan la capacidad de buscar y analizar información, 
desarrollar posturas, expresarse en forma fundada, deliberar, escuchar al otro, representar intereses de una colectividad, etcétera.

ii) Participación y toma de decisiones democráticas en forma vivencial. Algunos programas acentúan la vivencia de la participación democrática en su medio o en un medio «de laboratorio», proponiendo ideas frente al grupo, representando a otros o los propios intereses, participando en la decisión e implementación de actividades, liderando, resolviendo pacíficamente conflictos, con asertividad, cuidado de otros, etcétera.

iii) Sujetos de derechos y conciencia, con relaciones respetuosas de derechos. Algunos programas tienen como eje la promoción de derechos de los participantes, tales como el derecho a la cultura y recreación, a un ambiente no violento e igualdad de género, entre otros.

El desafío de los programas ha sido crear ambientes en que niños y jóvenes puedan tener una experiencia de aprendizaje positiva, en que se practiquen valores, ejerciten habilidades y se aprendan conocimientos acerca de la ciudadanía. Así, los programas comparten una práctica participativa e interactiva que enfatiza la idea del aprendizaje significativo y activo. De esta manera, se privilegia que los temas tratados o las situaciones representadas sean de interés para los niños, intentando acercar los contenidos a la realidad en que viven.

Algunos de estos programas se anclan en actividades establecidas en la institución — clases de historia o de orientación-, mientras que otros son pensados en un espacio extraescolar. Las oportunidades que los programas de formación ciudadana ofrezcan de establecer vínculos sociales y de conectarse con otros, así como la medida en que estos vínculos validen las capacidades de las personas, permitirán desarrollar un sentido de identidad colectiva que promueva los valores y actitudes de compromiso con otros y con el bienestar común.

Si bien existen programas en nuestro país que se encuentran trabajando en el desarrollo de estas competencias en niños y jóvenes, muchos deben acoger las necesidades y problemáticas que los mismos jóvenes visualizan en este campo. Sólo de esta manera se profundizará en los conocimientos, habilidades y actitudes que permitan a cada individuo ejercer su ciudadanía de manera activa, participativa, crítica, constructiva y responsable.

A su vez, se percibe que la solución o manera de enfrentar estos desafíos involucra distintos niveles de intervención. En esta misma línea, algunos deben ser abordados en los espacios en que se ejecutan 
estos programas, $\mathrm{y}$ otros a nivel de los organismos gubernamentales que intencionan la formación de estas competencias o a nivel social. Por eso, se requiere un trabajo colaborativo y coordinado de parte de los distintos actores involucrados.

SANTIAGO (CHILE), ABRIL 2010

RECIBIDO: ABRIL 2010

ACEPTADO: JUNIO 2010

\section{REFERENCIAS BIBLIOGRÁFICAS}

COMISIÓN ECONÓMICA PARA AMÉRICA LATINA Y EL CARIBE (CEPAL) (2000): Equidad, desarrollo y ciudadanía. Santiago: CEPAL.

Flanagan, C.; J. BOWES, B. JonsSON, B. CSAPO \& E. SHEBlanOva (1998): "Ties that bind: correlates of adolescence civic commitment in seven countries». Journal of Social Issues, 54 (3).

GONZÁLEZ, S. (2007): «La noción de ciudadanía en jóvenes estudiantes secundarios y universitarios: un análisis de estudios comparados de la nueva ciudadanía». En A. ZAMBRANO, G. ROZAS, I. MAgAÑA, D. Asún y R. PÉREZ-LuCO (editores): Psicología comunitaria en Chile: evolución, perspectivas y proyecciones. Santiago: RIL Editores.

JANOSKI, T. \& B. GRAN (2002): «Political citizenship: Foundations of rights». En ENGIN F. ISIN \& BRYAN S. TURNER (eds.): Handbook of Citizen Studies. London: Sage.

MARTÍNEZ K., M. (1999): «Comprensión de la cultura no ciudadana en Chile». En: Ciudadanía en Chile: el desafío cultural del nuevo milenio. Santiago: División de Organizaciones Sociales, Ministerio Secretaría General de Gobierno.

MARTÍNEZ, M. L.; C. SiLVA y A. C. HERNÁNDEZ (en revisión): «¿En qué ciudadanía creen los jóvenes? Aspiraciones de ciudadanía en jóvenes líderes y sus motivaciones para la participación social».

- - - M. CARMONA, P. CUMSILle y C. Flanagan (en revisión): «Young Chileans' views of citizenship: Findings from the first post-Pinochet generation».

MINEDUC (2005): «Informe comisión formación ciudadana». Santiago: MINEDUC. 
TORNEY-PURTA, J.; R. LEHMANN, H. OSWALD \& W. SCHULZ (2001): Citizenship and education in twentyeight countries: Civic knowledge and engagement at age fourteen. Amsterdam: International Association for the Evaluation of Educational Achievement.

TURNER, B. (1993): «Contemporary problems in the theory of citizenship». En B. TURNER (eds.): Citizenship and Social Theory. London: Sage. 